\title{
Analysis of detergent-resistant membranes associated with apical and basolateral GPI-anchored proteins in polarized epithelial cells
}

\author{
Simona Tivodar ${ }^{\mathrm{a}}$, Simona Paladino ${ }^{\mathrm{a}, \mathrm{b}}$, Rudolf Pillich ${ }^{\mathrm{a}}$, Alessandro Prinetti ${ }^{\mathrm{c}}$, Vanna Chigorno ${ }^{\mathrm{c}}$, \\ Gerrit van Meer ${ }^{\mathrm{d}}$, Sandro Sonnino ${ }^{\mathrm{c}}$, Chiara Zurzolo ${ }^{\mathrm{a}, \mathrm{e}, *}$ \\ a Dipartimento di Biologia e Patologia Cellulare e Molecolare, Università degli Studi di Napoli Federico II, via S. Pansini 5, 80131 Napoli, Italy \\ ${ }^{\mathrm{b}}$ CEINGE Biotecnologie Avanzate S.C.A.R.L., 80145 Napoli, Italy \\ ${ }^{\mathrm{c}}$ Center of Excellence on Neurodegenerative Diseases, Department of Medical Chemistry, Biochemistry and Biotechnology, University of Milan, \\ 20090 Segrate, Italy \\ ${ }^{\mathrm{d}}$ Department of Membrane Enzymology, Padualaan 8, 3584 CH, Utrecht University, The Netherlands \\ ${ }^{\mathrm{e}}$ Unité de Trafic Membranaire et Pathogénèse, Institut Pasteur, 25 rue du Docteur Roux, 75724 Paris Cedex 15, France
}

Received 27 July 2006; revised 4 September 2006; accepted 12 September 2006

Available online 20 September 2006

Edited by Felix Wieland

\begin{abstract}
Detergent-resistant membranes (DRMs) represent specialized membrane domains resistant to detergent extraction, which may serve to segregate proteins in a specific environment in order to improve their function. Segregation of glycosylphosphatidylinositol-anchored proteins (GPI-APs) in DRMs has been shown to be involved in their sorting to the apical membrane in polarized epithelial cells. Nonetheless, we have shown that both apical and basolateral GPI-APs associate with DRMs. In this report we investigated the lipid composition of DRMs associated with an apical and a basolateral GPI-AP.

We found that apical and basolateral DRMs contain the same lipid species although in different ratios. This specific lipid ratio is maintained after mixing the cells before lysis indicating that DRMs maintain their identity after Triton extraction. (C) 2006 Federation of European Biochemical Societies. Published by Elsevier B.V. All rights reserved.
\end{abstract}

Keywords: Rafts; Detergent-resistant membranes; Glycosylphosphatidylinositol-anchored proteins; Sorting; Polarity; Epithelial cells

\section{Introduction}

Lipid rafts constitute distinct liquid ordered phases enriched in sphingolipids and cholesterol that are resistant to extraction in cold detergents [1-3]. Because they segregate proteins and lipids in specific regions of the membranes, they have been implicated in several cell functions such as signalling and sorting [4]. One case in point is the one of glycosylphosphatidylinositol (GPI)-anchored proteins (GPI-APs) that are apically

\footnotetext{
${ }^{*}$ Corresponding author. Fax: +33 (0) 140613238.

E-mail addresses: zurzolo@pasteur.fr,

zurzolo@unina.it (C. Zurzolo).
}

Abbreviations: GPI-APs, glycosylphosphatidylinositol-anchored proteins; DRMs, detergent-resistant membranes; GH-DAF, growth hormone-decay accelerating factor; PLAP, placental alkaline phosphatase; SM, sphingomyelin; PC, phosphatidylcholine; PE, phosphatidyletanolamine; SL, sphingolipids; TX-100, Triton X-100 sorted in a range of polarized epithelial cell lines [2,5,6]. It has been proposed that GPI-APs are sorted to the apical surface via their incorporation in rafts [7]. However, the finding that in FRT cells GPI-APs are mainly delivered to the basolateral membrane [8,9] and that in MDCK cells both apical and basolateral GPI-APs associate with DRMs (detergent-resistant membranes) indicate that rafts are not sufficient to determine their apical sorting [10]. Furthermore, we have found that protein oligomerization, which could promote the stabilization of GPI-APs into rafts, is needed for their apical sorting [10]. The factors that promote protein oligomerization and apical sorting are still unclear. We consider two different hypotheses to be most likely: (1) the protein ectodomain may have a key role in determining first oligomerization and then stabilization into rafts, or (2) apical and basolateral proteins associate with different microdomains that would determine the rate of their clustering and therefore be responsible for the differential sorting. The latter hypothesis is supported by the fact that caveolae, which are specialized lipid microdomains, have been found enriched on the basolateral domain of several epithelial cell lines [11,12]. In addition, Thy-1 and PrPc, two GPI-APs respectively enriched in the axon and in the cell body of neurons, resembling the apical and basolateral domain of epithelial cells, associate with lipid microdomains of different composition [13].

In order to study the lipid composition of the apical and basolateral DRMs and to understand whether they maintain their specific composition after Triton extraction we analyzed the lipid fraction that co-immunoprecipitated with an apical (PLAP) and basolateral (GH-DAF) GPI-AP in DRMs from single or mixed FRT/PLAP and FRT/GHDAF clones.

\section{Material and methods}

\subsection{Reagents and antibodies}

Cell culture reagents were purchased from Gibco Laboratories (Grand Island, NY). The anti-PLAP antibody was from Rockland (Gilgertsville, PA), the anti-GH antibody was from Biotrend GMBH (Germany) and the anti-flotillin antibody was from BD Biosciences Pharmingen (San Jose, CA). Biotin and HRP-conjugated streptavidin were from Pierce (Rockford, IL). Protein G- and A-coupled magnetic beads (dynabeads) were from Dynal ASA (Oslo, Norway). All other reagents were purchased from Sigma Chemical Co. (St Louis, MO). 
[1- $\left.{ }^{3} \mathrm{H}\right]$-sphingosine (radiochemical purity over $98 \%$; specific radioactivity $2 \mathrm{Ci} / \mathrm{mmol}$ ) was prepared as previously described [14].

\subsection{Cell culture and transfections}

FRT cells were grown in F12 Coon's medium containing 5\% FBS and stably transfected with GH-DAF cDNA as previously described [15]. FRT clones expressing PLAP were previously obtained [9]. Surface expression of transfected GPI-APs was analyzed by confocal microscopy (Zeiss 510 LSM) and by biotinylation of cells grown on filters for 4 days as previously described [10].

\subsection{Assays for DRM association}

2.3.1. Triton $X-100(T X-100)$ extraction. Cells grown to confluency from one $60-\mathrm{mm}$ dish $\left(\sim 2 \times 10^{6}\right)$ were washed twice with PBS containing $1 \mathrm{mM} \mathrm{CaCl}{ }_{2}$ and $1 \mathrm{mM} \mathrm{MgCl}$ and then lysed for $20 \mathrm{~min}$ on ice in $1 \mathrm{ml} \mathrm{TNE} / \mathrm{TX}-100$ buffer $(25 \mathrm{mM}$ Tris- $\mathrm{HCl}, \mathrm{pH} 7.5,150 \mathrm{mM} \mathrm{NaCl}$, $5 \mathrm{mM}$ EDTA, $1 \%$ TX-100) and separated by centrifugation into soluble and insoluble fraction as previously described [2]

2.3.2. Sucrose density gradients. Cells that had just reached confluency in 150 -mm dishes $\left(20-25 \times 10^{6}\right)$ were subject to ultracentrifugation on discontinuous sucrose gradients as previously described [16]. Briefly, cells harvested in PBS containing $0.4 \mathrm{mM} \mathrm{Na} \mathrm{VO}_{4}$ were suspended in $1 \mathrm{ml}$ lysis buffer on ice (1\% TX-100, $10 \mathrm{mM}$ Tris buffer, pH 7.5, $150 \mathrm{mM} \mathrm{NaCl}, 5 \mathrm{mM}$ EDTA, $1 \mathrm{mM} \mathrm{Na} \mathrm{VO}_{4}, 1 \mathrm{mM}$ PMSF and $75 \mathrm{mU} / \mathrm{ml}$ aprotinin), Dounce homogenized. Post-nuclear supernatants ( $\sim 8 \mathrm{mg}$ proteins) were mixed with an equal volume of $85 \%$

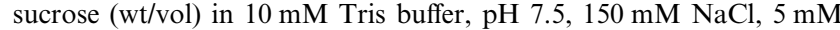
EDTA, $1 \mathrm{mM} \mathrm{Na} \mathrm{VO}_{4}$, and run on discontinuous sucrose gradients $(30-5 \%)$ at $200000 \times g$ for $17 \mathrm{~h}$ at $4{ }^{\circ} \mathrm{C}$. Eleven $1 \mathrm{ml}$ fractions were collected starting from the top of the tube. Fraction 5, located at the interface between $5 \%$ and $30 \%$ sucrose, was regarded as the sphingolipid-enriched membrane fraction.
A
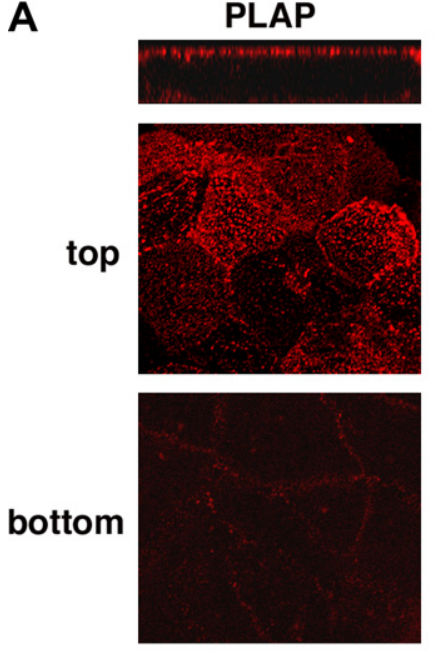

B

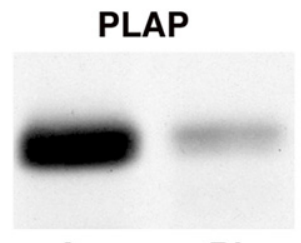

Ap
BI
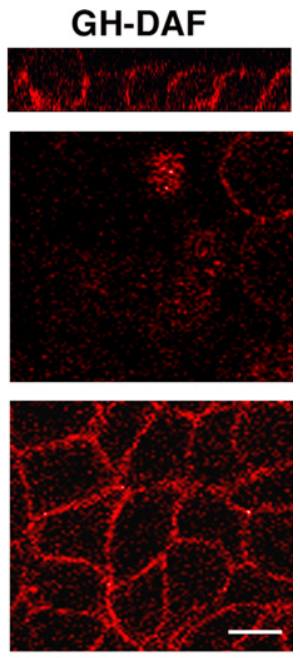

GH-DAF

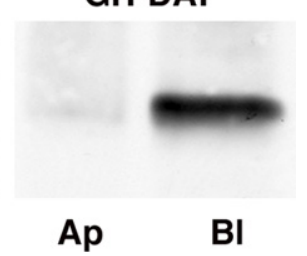

Fig. 1. PLAP and GH-DAF are differently sorted in FRT cells. PLAP or GH-DAF FRT clones were grown to confluency on filters, fixed and stained with specific antibodies against PLAP and GH followed by TRITC-conjugated secondary antibody in non-permeabilized conditions. Serial confocal sections were collected from the top to the bottom of cell monolayers (A). LC-biotin was added to the apical or the basolateral surface of FRT clones. Biotinylated proteins were immunoprecipitated using specific antibodies to PLAP or GH and revealed with HRP-conjugated streptavidin (B). Bar $=10 \mu \mathrm{m}$.
2.4. $\left[{ }^{3} H\right]$-sphingosine labelling and lipid analysis

Cells from two $150-\mathrm{mm}$ dishes $\left(40-50 \times 10^{6}\right)$ were incubated in the presence of $4 \times 10^{-8} \mathrm{M}\left[1-{ }^{3} \mathrm{H}\right]$-sphingosine $(7 \mathrm{ml} /$ dish) in culture medium for $2 \mathrm{~h}$ and then chased for $48 \mathrm{~h}$ in fresh culture medium [16]. The radioactivity associated with cells and with cell fractions was determined by liquid scintillation counting. Labelled samples were dialyzed and lyophilized. $\left[{ }^{3} \mathrm{H}\right]$ lipids were extracted twice with $0.4 \mathrm{ml}$ chloroform/methanol 2:1 (v/v), separated by mono-dimensional HPTLC using the solvent system chloroform/methanol/0.2\% acqueous $\mathrm{CaCl}_{2}$ 55:45:10 (v/v/v), revealed by digital autoradiography (250 dpm applied on a $3 \mathrm{~mm}$ line) with a Beta-Imager 2000 instrument, and analyzed by $\beta$-Vision software (Biospace, Paris) [16]. Cholesterol was quantified after separation on HPTLC by visualization with $15 \%$ concentrated sulphuric acid in 1-butanol [16]. Phosphatidylcholine was separated by a two-run mono-dimensional HPTLC using the solvent system chloroform/methanol 9:1 (v/v), followed by the solvent system chloroform/methanol/acid acetic/water 30:20:2:1 (v/v/v/v) and quantified after separation on HPTLC followed by specific detection with a molybdate reagent. Cholesterol and phosphatidylcholine were quantified by densitometry and comparison with known amounts of standard lipids (Molecular Analyst program, Bio-Rad Laboratories).

\subsection{Immunoprecipitation}

Cells grown to confluency from two 150 -mm dishes $\left(40-50 \times 10^{6}\right)$ were labelled with $\left[1-{ }^{3} \mathrm{H}\right]$-sphingosine and subject to ultracentrifugation on discontinuous sucrose gradients as aforementioned. Aliquots of fraction $5(800 \mu \mathrm{l})$ diluted with $200 \mu \mathrm{l}$ lysis buffer $(5 \%$ TX-100, $10 \mathrm{mM}$ Tris buffer, pH 7.5, $150 \mathrm{mM} \mathrm{NaCl}, 5 \mathrm{mM}$ EDTA, $1 \mathrm{mM}$ $\mathrm{Na}_{3} \mathrm{VO}_{4}, 1 \mathrm{mM}$ PMSF and $75 \mathrm{mU} / \mathrm{ml}$ aprotinin) were precleared twice with dynabeads for $2 \mathrm{~h}$ and incubated overnight at $4{ }^{\circ} \mathrm{C}$ with antiPLAP or anti-GH antibodies. Immunoprecipitates were recovered using protein A- or protein G-coupled magnetic beads [16]. Lipids
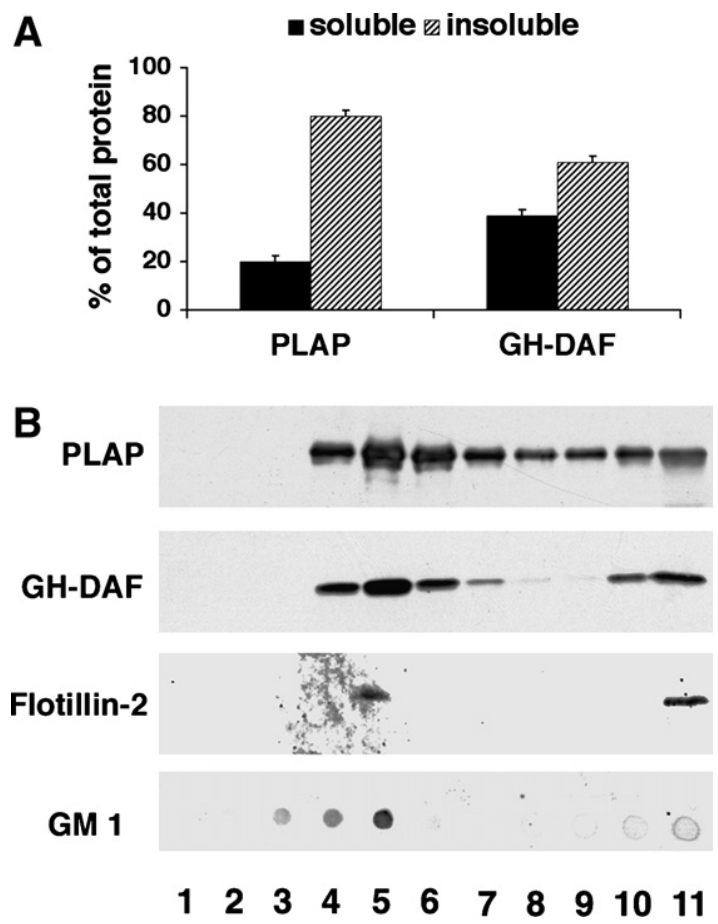

Fig. 2. Both PLAP and GH-DAF associate with DRMs. PLAP or GH-DAF clones were lysed in TNE/TX-100 buffer at $4{ }^{\circ} \mathrm{C}$ and separated by centrifugation into insoluble and soluble fractions. Percentages of insoluble and soluble proteins in TX-100 from three experiments are shown. Errors bars are indicated (A). Eleven $1 \mathrm{ml}$ fractions were collected from top (1) to bottom (11) from sucrose density gradients. Proteins were analyzed by SDS-PAGE and detected by Western blotting using specific anti-PLAP, anti-GH and antiflotillin-2 antibodies. An aliquot of each fraction was spotted on the nitrocellulose membrane and $\mathrm{GM}_{1}$ was revealed using cholera-toxin conjugated to HRP (B). 
were extracted from the immunoprecipitates and analyzed as described above. $1 / 5$ of the samples were analyzed by SDS-PAGE.

\section{Results}

\subsection{Analysis of surface distribution and DRM association of PLAP and GH-DAF in FRT cells}

The surface distribution of PLAP (placental alkaline phosphatase, a native GPI-AP) and GH-DAF (in which growth hormone is fused with the GPI-attachment signal from DAF) was analyzed by immunofluorescence and confocal microscopy in stably transfected FRT cells grown in polarized conditions on transwell filters (Fig. 1A). These experiments confirmed that PLAP was preferentially localized on the apical domain [9], while GH-DAF was preferentially localized on the basolateral domain, in agreement with what has been shown in MDCK cells [10]. By surface biotinylation we quantified the surface distribution of the two proteins that was respectively
A

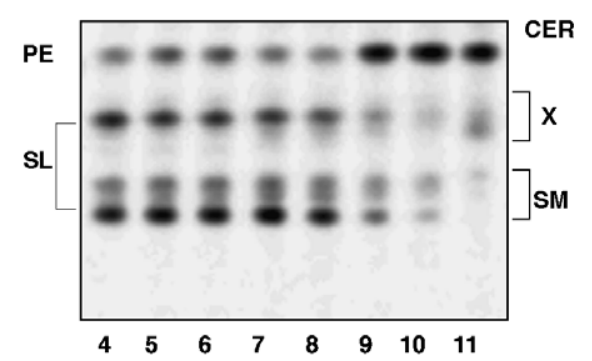

B

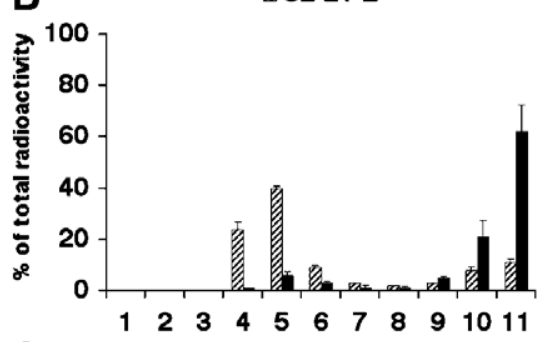

C

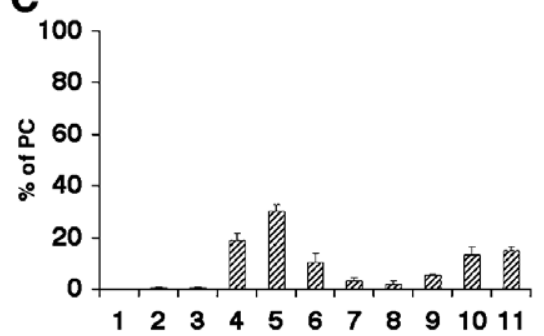

D
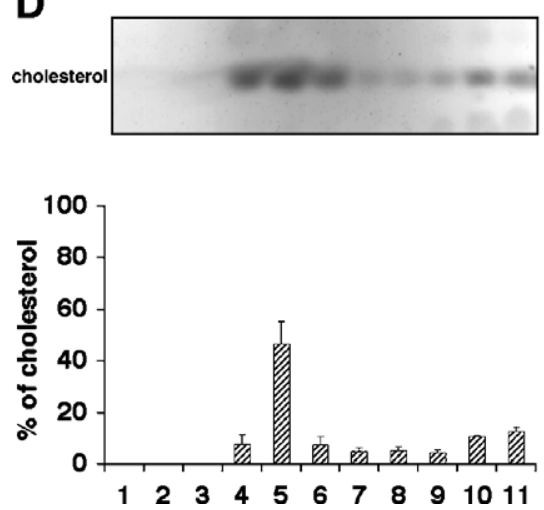

\section{GH-DAF}

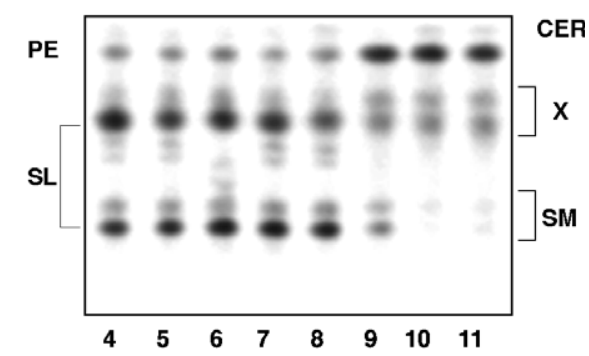

凤SL $\square$ PE
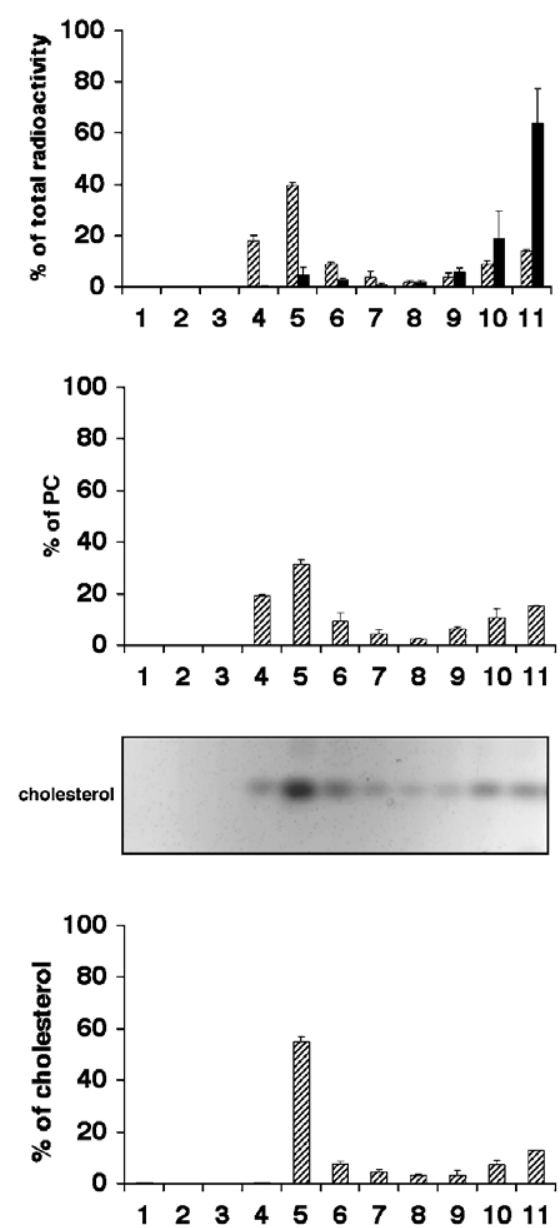

Fig. 3. Lipid distribution on sucrose density gradients. PLAP or GH-DAF clones were labelled with $\left[{ }^{3} \mathrm{H}\right]$-sphingosine, lysed in TNE/TX-100 at $4{ }^{\circ} \mathrm{C}$ and run on sucrose density gradients. Lipids from each of the 11 fractions were extracted in chloroform/methanol. $250 \mathrm{dpm} / \mathrm{fraction}$ was separated by HPTLC and detected by autoradiography (A). We have indicated with $\mathrm{X}$ the unknown species of sphingolipids labelled with [ $\left.{ }^{3} \mathrm{H}\right]$-sphingosine. Quantitation of sphingolipid and PE radioactivity distribution within the gradient fractions. Data are expressed as \% of total radioactivity associated with each lipid present in homogenate (B). The distribution of PC and cholesterol, measured by colorimetric assays, are respectively shown in (C) and (D). Data are expressed as \% of each lipid present in homogenate. Data are the means of three independent experiments \pm S.D. 
A

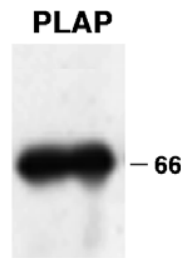

F5
GH-DAF

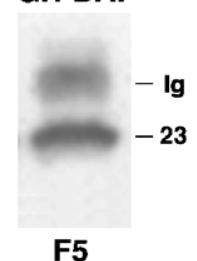

\section{B}

PLAP

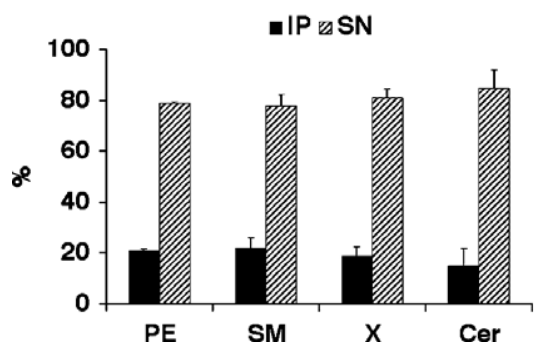

GH-DAF

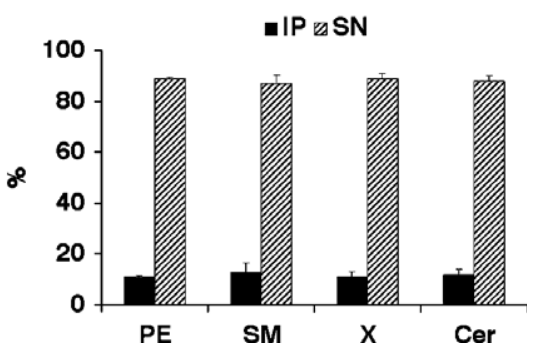

Fig. 4. Lipid analysis of DRMs associated with PLAP or GH-DAF. PLAP or GH-DAF clones were labelled with [ $\left.{ }^{3} \mathrm{H}\right]$-sphingosine, lysed in TNE/ TX-100 at $4{ }^{\circ} \mathrm{C}$ and purified by centrifugation to equilibrium on sucrose density gradients. Fraction 5 was immunoprecipitated using either PLAP or GH-DAF antibodies. Proteins (1/5 of immunoprecipitate) were run on SDS-PAGE and revealed by Western blotting (A). Lipids associated with immunoprecipitates (IP) were extracted in chloroform/methanol and separated by HPTLC. The quantitation of radioactive lipids (SM, Cer, X, PE) is shown in (B). For each lipid species values are expressed as percent of radioactivity present in immunoprecipitates and in the supernatant (SN) (B). Data are the means of three independent experiments \pm S.D.

Table 1

Distribution of radioactivity after immunoprecipitation with anti-PLAP or anti-GH antibodies in sphingolipid-enriched membrane fractions from FRT cells in culture

\begin{tabular}{|c|c|c|c|c|c|}
\hline & Total & IP & $\%$ in IP & Preclear & Supernatant \\
\hline FRT cells expressing PLAP (IP $\alpha$ PLAP) & 243100 & 46100 & $19 \pm 0.6$ & 19500 & 177500 \\
\hline FRT cells expressing GH-DAF (IP $\alpha \mathrm{GH}$ ) & 257500 & 28200 & $11 \pm 1.5$ & 22000 & 207300 \\
\hline Mixed cells (IP $\alpha$ PLAP) & 257000 & 34900 & $13 \pm 1$ & 16100 & 205700 \\
\hline Mixed cells (IP $\alpha \mathrm{GH}$ ) & 257000 & 17100 & $7 \pm 0.7$ & 22400 & 217500 \\
\hline
\end{tabular}

Cell lipids were previously metabolically labelled with $\left[{ }^{3} \mathrm{H}\right]$-sphingosine. Data are expressed in dpm. Percentages represent values of three independent experiments \pm S.D.

Table 2

Percent of spingosine-labelled lipid species in the anti-PLAP or anti$\mathrm{GH}$ immunoprecipitates of fraction 5

\begin{tabular}{lllll}
\hline & PE & SM & X & Cer \\
\hline FRT cells expressing & $22 \pm 1$ & $63 \pm 3.6$ & $12 \pm 3.2$ & $3 \pm 0.6$ \\
$\quad$ PLAP (IP $\alpha$ PLAP) & & & & \\
FRT cells expressing & $20 \pm 2$ & $64 \pm 1.5$ & $13 \pm 3.2$ & $3 \pm 1$ \\
$\quad$ GH-DAF (IP $\alpha \mathrm{GH})$ & & & & \\
Mixed cells (IP $\alpha$ PLAP) & $14 \pm 2.3$ & $62 \pm 1$ & $21 \pm 3.2$ & $3 \pm 1$ \\
Mixed cells (IP $\alpha \mathrm{GH})$ & $14 \pm 2$ & $63 \pm 2.6$ & $20 \pm 2$ & $3 \pm 1$ \\
\hline
\end{tabular}

Percentages represent values of three independent experiments \pm S.D. $\sim 90 \%$ apical for PLAP and $\sim 99 \%$ basolateral for GH-DAF (Fig. 1B).

In order to analyze the amount of PLAP and GH-DAF associated with DRMs we performed TX-100 extraction and flotation on sucrose density gradients, as previously described $[2,10,16]$. At steady state both proteins (respectively $\sim 80 \%$ of total PLAP and $\sim 61 \%$ of total GH-DAF) were insoluble in TX-100 (Fig. 2A). After centrifugation to equilibrium on sucrose density gradients both proteins peaked in fraction 5 at the interface between $5 \%$ and $30 \%$ sucrose which is the most representative fraction of DRMs [16], as shown by the flota-

Table 3

Distribution of cholesterol after immunoprecipitation with anti-PLAP or anti-GH antibodies in sphingolipid-enriched membrane fractions from labelled FRT cells in culture

\begin{tabular}{|c|c|c|c|c|c|}
\hline & Total & IP & $\%$ in IP & Preclear & Supernatant \\
\hline FRT cells expressing PLAP (IP $\alpha$ PLAP) & 164.0 & 24.5 & $15 \pm 1$ & n.a & 123.3 \\
\hline FRT cells expressing GH-DAF (IP $\alpha \mathrm{GH})$ & 184.0 & 16.6 & $9 \pm 2.5$ & n.a & 151.1 \\
\hline Mixed cells (IP $\alpha$ PLAP) & 168.0 & 20.7 & $12 \pm 2$ & n.a & 133.0 \\
\hline Mixed cells (IP $\alpha \mathrm{GH}$ ) & 168.0 & 11.1 & $7 \pm 1$ & n.a & 142.5 \\
\hline
\end{tabular}

Data are expressed in nmol. n.a., not analyzed. Percentages represent values of three independent experiments \pm S.D. 
tion profiles of flotillin-2 and $\mathrm{GM}_{1}$ used as control bona-fide DRM markers (Fig. 2B). In contrast to the majority of the proteins in the cell lysates that accumulated in the soluble fractions (8-11) (data not shown) about $63 \%$ of PLAP and $68 \%$ of GH-DAF were enriched in fractions 4-6 (Fig. 2B), thus indicating that both apical and basolateral GPI-APs are associated with DRMs.

\subsection{Analysis of the lipid composition of DRMs in PLAP or GH-DAF expressing FRT cells}

In order to understand whether the DRM composition was affected by the exogenous expression of apical or basolateral GPI-APs, we analyzed the lipid composition of the DRM fraction in FRT cells expressing either PLAP or GH-DAF. Cells were labelled with $\left[1-{ }^{3} \mathrm{H}\right]$-sphingosine, which is converted into more complex sphingolipids, while the catabolic tritiated ethanolamine fragment is recycled for the biosynthesis of phosphatidylethanolamine (PE), a glycerophospholipid that is a marker of non-DRM fractions [16,17]. The radioactive lipid pattern was determined by HPTLC and radioimaging of the 11 fractions collected from sucrose density gradient centrifugation (Fig. 3A). Sphingolipids (SL) (sphingomyelin (SM) and the unknown $\mathrm{X}$ species of sphingolipids labelled with $\left[1-{ }^{3} \mathrm{H}\right]-$ sphingosine) were highly enriched in fraction 5 (Fig. 3B), while the majority of PE was found, as expected, in the soluble fractions of the sucrose density gradients in both the cell lines (Fig. 3B).

Phosphatidylcholine (PC) analyzed by HPTLC and a colorimetric assay was also quite enriched in fraction 5 from both clones (Fig. 3C), as previously described [16,17]. Finally, $\sim 42 \mathrm{nmol} / \mathrm{mg}$ protein of cholesterol was found in the post-nuclear supernatants and about $50 \%$ of it was associated with fraction 5 in both FRT clones (Fig. 3D). Thus these data show that the lipid distribution along sucrose density gradients is similar in both clones (Fig. 3) and is not affected by protein expression.

\subsection{Lipid analysis of DRM fractions associated with PLAP or $G H-D A F$}

To analyze whether apical and basolateral GPI-APs associated to similar DRMs we specifically immunoprecipitated each protein from fraction 5 of the gradients after $\left[{ }^{3} \mathrm{H}\right]$-sphingosinelabelling (Fig. 4A). About 19\% and 11\%, respectively, of the total radioactivity present in fraction 5 was recovered respectively in the PLAP or GH-DAF immunoprecipitates, representing co-immunoprecipitated labelled sphingolipids (Table 1). After chloroform/methanol 2:1 (v/v) extraction, HPTLC and digital autoradiography $\sim 22 \%$ of SM, $\sim 15 \%$ of Cer and $\sim 21 \%$ of PE were associated with PLAP immunoprecipitates while $\sim 13 \%$ of SM, $\sim 12 \%$ of Cer and $\sim 11 \%$ of PE were found in the GH-DAF immunoprecipitates (Fig. 4B). The relative percentage of each type of labelled lipid in PLAP and GH immunoprecipitates of fraction 5 was very similar (Table 2). The total lipid extracts of the immunoprecipitates were then analyzed for cholesterol $(\sim 24.5 \mathrm{nmol}$ for PLAP and $\sim 16.6$ nmol for GH-DAF) (Table 3$)$ and for PC ( $19 \%$ for PLAP and $\sim 6 \%$ for GH-DAF) (Table 4 ). The ratio between [ $\left.{ }^{3} \mathrm{H}\right]-\mathrm{SM}$ :cholesterol:PC was 2663:2.24:1 (dpm:nmol:nmol) for PLAP and 4346:4.48:1 (dpm:nmol:nmol) for GH-DAF indicating that there were no significant qualitative differences in the lipid composition of DRMs associated with the apical or basolateral GPI-AP. However, the calculation of the relative lipid enrichment (SL or cholesterol over PC) clearly shows that DRMs associated with basolateral GH-DAF contain higher amounts of SL and cholesterol with respect to apical PLAP (Table 5).

\subsection{Effect of $T X-100$ on DRM formation}

To rule out the possibility that TX-100 had an effect in mixing apical and basolateral DRMs we performed TX-100 extraction and separation on sucrose density gradients after mixing the two cell lines previously labelled with $\left[{ }^{3} \mathrm{H}\right]$-sphingosine. As shown in Fig. 5 the distribution of proteins (Fig. 5A), radioactive lipids (Fig. 5B), PC (Fig. 5C) and cholesterol (Fig. 5D) after separation of the mixed lysates on sucrose density gradients was similar to that from gradients derived from the single cell line extracts (compare Fig. 5 with Fig. 3).

Fraction 5 was then immunoprecipitated using either antiPLAP or anti-GH antibodies and proteins were analyzed by SDS-PAGE and Western blotting (Fig. 6A). In these mixed lysates GH-DAF was not co-immunoprecipitated with the antiPLAP antibody and vice-versa (Fig. 6A), therefore indicating that the addition of the detergent does not lead to a mixing of the two proteins or their surrounding environments. As expected about half of the total lipid radioactivity was recovered in the mixed compared to the single lysate immunoprecipates ( $\sim 13 \%$ for PLAP and $\sim 7 \%$ for GH-DAF) (Table 1$)$. As shown in Fig. $6 \mathrm{~B} \sim 17 \%$ of $\mathrm{SM}, \sim 15 \%$ of Cer and $\sim 10 \%$ of $\mathrm{PE}$ was associated with PLAP immunoprecipitates while $\sim 8 \%$ (SM), $\sim 7 \%$ (Cer) and $\sim 5 \%$ (PE) were recovered in the GH immunoprecipitates. Thus the relative percentage of each type of radiolabelled lipid immunoprecipitated either with anti-PLAP or

Table 5

Relative enrichment of sphingolipids and cholesterol over phosphatidylcholine (PC) in the anti-PLAP or anti-GH immunoprecipitates

\begin{tabular}{lll}
\hline & IP single cells & IP mixed cells \\
\hline $\begin{array}{ll}(\text { Sphingolipids/PC })_{\text {DAF }} \\
\text { (Sphingolipids/PC })_{\text {PLAP }}\end{array}$ & 1.8 & 1.6 \\
$\frac{(\text { cholesterol/PC })_{\text {DAF }}}{(\text { cholesterol/PC })_{\text {PAAP }}}$ & 1.9 & 1.8 \\
\hline
\end{tabular}

Table 4

Distribution of phosphatidylcholine after immunoprecipitation with anti-PLAP or anti-GH antibodies in sphingolipid-enriched membrane fractions from labelled FRT cells in culture

\begin{tabular}{|c|c|c|c|c|c|}
\hline & Total & IP & $\%$ in IP & Preclear & Supernatant \\
\hline FRT cells expressing PLAP (IP $\alpha$ PLAP) & 57.6 & 10.9 & $19 \pm 2$ & n.a. & 41.7 \\
\hline FRT cells expressing GH-DAF (IP $\alpha \mathrm{GH}$ ) & 62.0 & 3.7 & $6 \pm 0.7$ & n.a. & 53.3 \\
\hline Mixed cells (IP $\alpha$ PLAP) & 57.8 & 5.1 & $9 \pm 1.4$ & n.a. & 47.8 \\
\hline Mixed cells (IP $\alpha \mathrm{GH}$ ) & 57.8 & 1.7 & $3 \pm 0.7$ & n.a. & 48.9 \\
\hline
\end{tabular}

Data are expressed in nmoles. n.a., not analyzed. Percentages represent values of two independent experiments \pm S.D. 


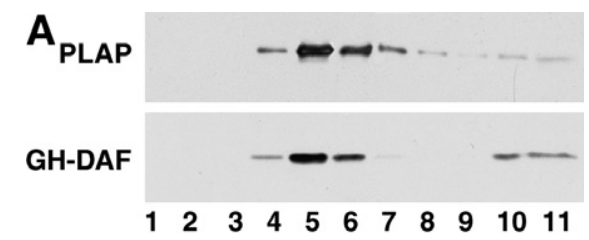

B
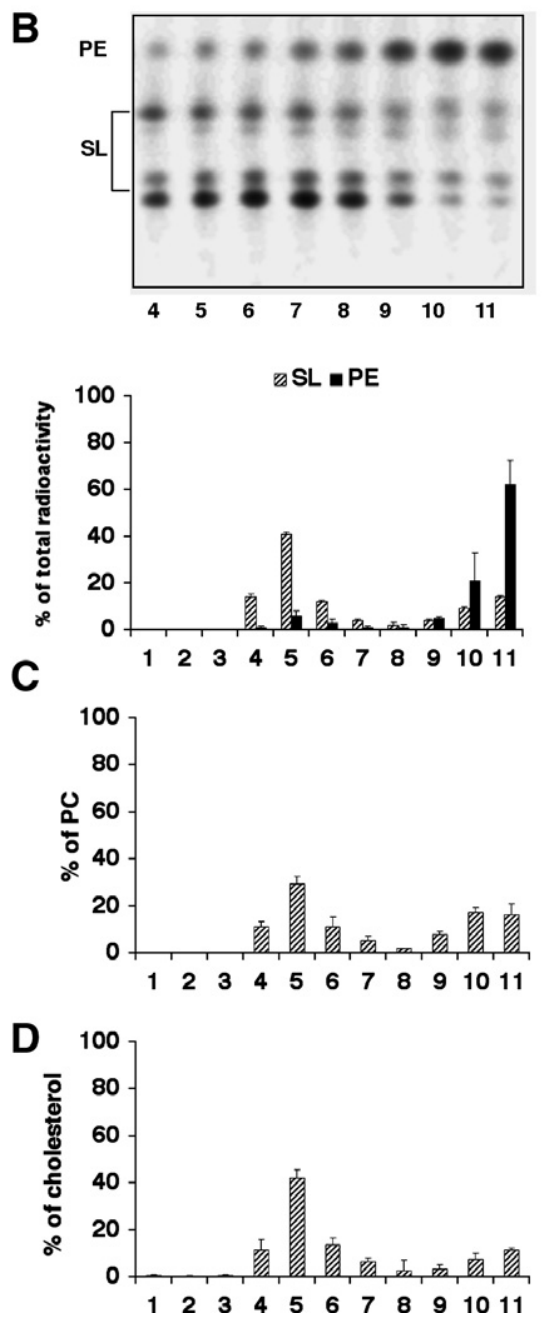

Fig. 5. Protein and lipid distribution on sucrose density gradients from mixed lysates of cells expressing PLAP and GH-DAF. PLAP and GHDAF clones (plated in the same number) were labelled with $\left[{ }^{3} \mathrm{H}\right]-$ sphingosine, harvested, mixed together and lysed in TNE/TX-100 buffer at $4{ }^{\circ} \mathrm{C}$. TX-100 extracts were purified by centrifugation to equilibrium on sucrose density gradients. Proteins were run on SDSPAGE and PLAP and GH-DAF were detected by Western blotting (A). Lipids were extracted and detected as described in Fig. 3. The distribution of sphingosine-labelled species on sucrose density gradients and their quantitation are shown in (B) (upper and lower panel, respectively). PC and cholesterol distributions are shown in (C) and (D), respectively. Data are expressed as \% of each lipid present in the homogenate. Data are the means of two independent experiments \pm S.D.

anti-GH remained constant between single and mixed lysates (Table 2). Interestingly, we observed a higher cholesterol recovery, both in PLAP $(\sim 20 \mathrm{nmol})$ and $\mathrm{GH}(\sim 11 \mathrm{nmol})$ immunoprecipitates, from the mixed lysates (Table 3 ). Like for SL the amount of PC recovered in the immunoprecipitates of fraction 5 from the mixed lysates was about half of the amount in the single lysates (Table 4). The ratio $\left[{ }^{3} \mathrm{H}\right]-\mathrm{SM}$ : cholesterol:PC, in the immunoprecipitates was respectively 3700:4.05:1 (dpm:nmol:nmol) for PLAP, and 5335:6.5:1 (dpm:nmol:nmol) for GF-DAF, similar to the ratio found in the immunoprecipitates from single cell lysates (Table 5). These results confirmed that the composition of DRMs associated with an apical and a basolateral GPI-AP are qualitatively very similar and showed that the relative enrichment of the different lipid species was maintained in the mixed lysates compared to single lysate (Table 5).

\section{Discussion}

We have investigated here the lipid composition of DRMs associated with PLAP and GH-DAF, respectively sorted to the apical and basolateral domains of FRT cells, in order to understand whether apical and basolateral GPI-APs are surrounded by a different lipid environment and whether this could be linked to their different sorting. We found that PLAP and GH-DAF were similarly distributed in a 2-step sucrose density gradient (Fig. 2) and were enriched in fraction 5 to similar extents $(\sim 30 \%)$. This fraction contains $\sim 40 \%$ of cellular sphingolipids, $\sim 50 \%$ of cellular cholesterol and $\sim 30 \%$ of cellular PC, but only $5 \%$ of cellular PE (Fig. 3), clearly indicating a lipid segregation in the different gradient fractions. These results support the increasing evidence for the existence of specialized lipid domains in the membrane $[2,16]$ and indicate that the lipid distribution found in FRT cells is similar to that recovered from other cell lines $[2,16,18]$. Our data also show that DRM-fractions derived from different FRT clones are similar, therefore indicating that the expression of different GPI-APs does not alter lipid extraction nor their distribution on sucrose gradients.

After labelling the cells with $\left[1-{ }^{3} \mathrm{H}\right]$-sphingosine we analyzed the lipids co-immunoprecipitated with PLAP and GH-DAF from the DRM fraction of sucrose gradients. We found no qualitative differences in the lipid species co-purified with apical or basolateral GPI-APs (Fig. 4). However, a different ratio between the lipid species was consistently observed in different experiments and was maintained after mixing the two FRT clones before lysis (Fig. 6), thus demonstrating that there is no artificial lipid mixing or domain formation caused by TX100 extraction and suggesting that the co-immunoprecipitated lipids represent the boundary lipids around each protein [3]. Specifically we found an enrichment of SL and cholesterol over PC in the DRM fraction co-immunoprecipitated with basolateral GH-DAF compared to the ones co-immunoprecipitated with apical PLAP (Table 5). These data are in agreement with recent data demonstrating a twofold increase in the cholesterol:phospholipids ratio in the basolateral membrane of guinea pig colonic epithelia compared to the apical one [19]. Cholesterol plays an important role as a linker molecule [3], but it can also cause or enhance lateral separation of lipids, depending on its concentration. Higher cholesterol in the epithelial basolateral membrane leads to higher permeability compared to the apical one that needs to be better protected from the external aggression [19]. Furthermore, a high amount of cholesterol could lead to greater spacing between basolateral GPI-APs, therefore impairing the possibility for them to come into closer contact with each other and ultimately 

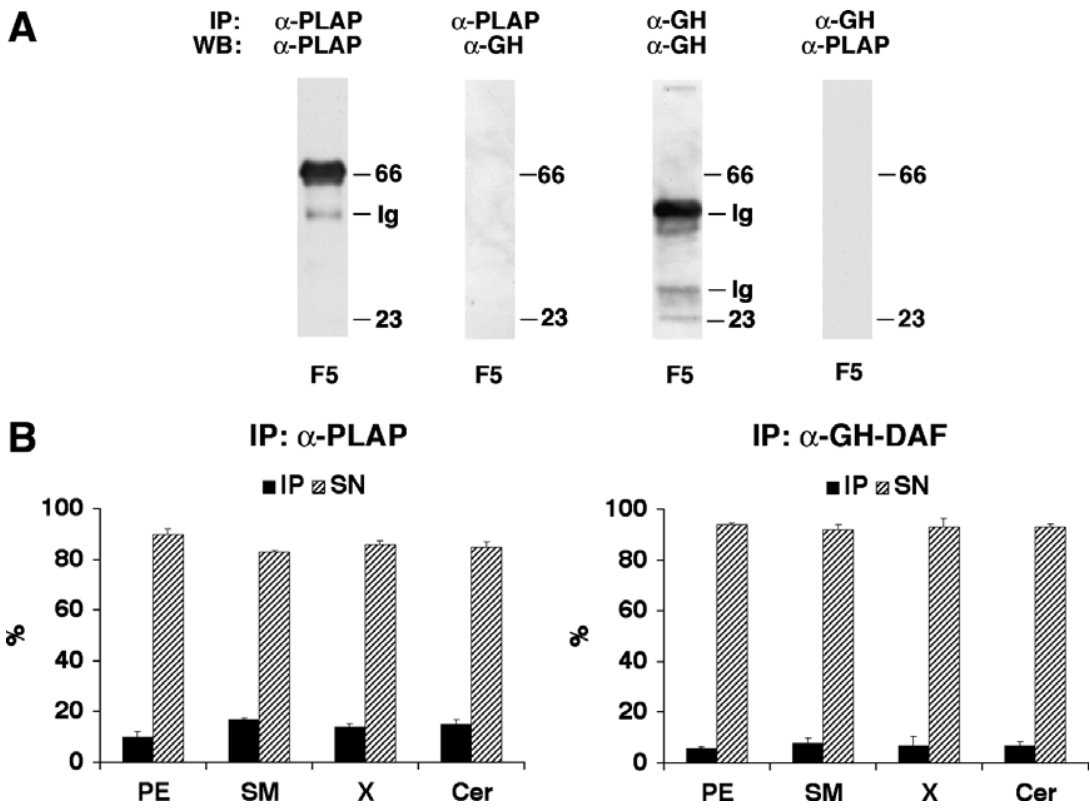

Fig. 6. Lipid analysis of DRMs associated with PLAP- or GH-DAF-associated DRMs derived from mixed cell lysates. FRT cells expressing PLAP and GH-DAF were labelled with $\left[{ }^{3} \mathrm{H}\right]$-sphingosine, harvested, mixed together and lysed in TNE/TX-100 buffer at $4{ }^{\circ} \mathrm{C}$. TX-100 extracts were purified by centrifugation to equilibrium on the sucrose gradients. Fraction 5 was immunoprecipitated using antibodies against PLAP or GH-DAF. Proteins (1/5 of immunoprecipitate) were run on SDS-PAGE and revealed by Western blotting using specific anti-PLAP or anti-GH antibodies (A). The molecular weights of PLAP $(66 \mathrm{kDa})$ and GH-DAF $(23 \mathrm{kDa})$ and immunoglobulins $(\mathrm{Ig})$ are indicated. Lipids were extracted and detected as described in Fig. 3. The distribution of radioactive lipids (SM, Cer, X, PE) is shown. For each lipid species values are expressed as percents of radioactivity present in immunoprecipitates (IP) and in the supernatants (SN) (B).

impairing their oligomerization, a process that we have shown to be necessary for apical GPI-APs sorting [10]. The glycolipid anchor of GPI-APs could also have an active role in this process by promoting a different affinity for different kinds of lipid microdomains. Consequently, if a GPI-AP is in a favorable lipid environment for oligomerization, then it could be apically sorted. Affinity for lipid rafts and oligomerization could work synergistically to promote apical sorting of GPI-APs. At this stage we cannot conclude whether the quantitative differences that we have found in the lipid composition of DRMs associated with apical and basolateral GPI-APs have a direct role in apical sorting, but our results will allow further analysis that will aim to address the role of the proteinaceus ectodomain and the role of the GPI-anchor. Only when all these additional possibilities have been explored will we be able to have a complete picture of the mechanism and of the different factors involved in apical sorting of GPI-APs.

Acknowledgments: This work was supported by COFIN-PRIN (2002, 2003, 2004), FIRST $(2002,2003,2004)$ and FIRB (2001) to A.P, V.C. and S.S.; by COFIN-PRIN (2004), FIRB (2003) and ANR (05-BLAN 296-01) to C.Z. and E.U. (HPRN-CT-2000-00077) to GvM and C.Z. S.T. and R.P. received fellowships by EU training student grant (HPRN-CT-2000-00077).

\section{References}

[1] Brown, D.A. and London, E. (2000) Structure and function of sphingolipid- and cholesterol-rich membrane rafts. J. Biol. Chem. 275, 17221-17224.

[2] Brown, D.A. and Rose, J.K. (1992) Sorting of GPI-anchored proteins to glycolipid-enriched membrane subdomains during transport to the apical cell surface. Cell 68, 533-544.
[3] Simons, K. and Vaz, W.L. (2004) Model systems, lipid rafts, and cell membranes. Annu. Rev. Biophys. Biomol. Struct. 33, 269 295.

[4] Simons, K. and Toomre, D. (2000) Lipid rafts and signal transduction. Nat. Rev. Mol. Cell Biol. 1, 31-39.

[5] Lisanti, M.P., LeBivic, A., Saltiel, A.R. and Rodriguez-Boulan, E. (1990) Preferred apical distribution of glycosyl-phosphatidylinositol (GPI) anchored proteins: a highly conserved feature of the polarized epithelial cell phenotype. J. Membr. Biol., 113155113167.

[6] Rodriguez-Boulan, E., Kreitzer, G. and Musch, A. (2005) Organization of vesicular trafficking in epithelia. Nat. Rev. Mol. Cell Biol. 6, 233-247.

[7] Simons, K. and Ikonen, E. (1997) Functional rafts in cell membranes. Nature 387, 569-572.

[8] Sarnataro, D., Paladino, S., Campana, V., Grassi, J., Nitsch, L. and Zurzolo, C. (2002) PrPC is sorted to the basolateral membrane of epithelial cells independently of its association with rafts. Traffic 3, 810-821.

[9] Lipardi, C., Nitsch, L. and Zurzolo, C. (2000) Detergent-insoluble GPI-anchored proteins are apically sorted in Fischer rat thyroid cells, but interference with cholesterol or sphingolipids differentially affects detergent insolubility and apical sorting. Mol. Biol. Cell 11, 531-542.

[10] Paladino, S., Sarnataro, D., Pillich, R., Tivodar, S., Nitsch, L. and Zurzolo, C. (2004) Protein oligomerization modulates raft partitioning and apical sorting of GPI-anchored proteins. J. Cell Biol. 167, 699-709.

[11] Vogel, U., Sandvig, K. and van Deurs, B. (1998) Expression of caveolin-1 and polarized formation of invaginated caveolae in Caco-2 and MDCK II cells. J. Cell Sci. 111 (Pt 6), 825-832.

[12] Lahtinen, U., Honsho, M., Parton, R.G., Simons, K. and Verkade, P. (2003) Involvement of caveolin-2 in caveolar biogenesis in MDCK cells. FEBS Lett. 538, 85-88.

[13] Brugger, B., Graham, C., Leibrecht, I., Mombelli, E., Jen, A., Wieland, F. and Morris, R. (2004) The membrane domains occupied by glycosylphosphatidylinositol-anchored prion protein and Thy-1 differ in lipid composition. J. Biol. Chem. 279, 75307536. 
[14] Tettamanti, G., Bonali, F., Marchesini, S. and Zambotti, V. (1973) A new procedure for the extraction, purification and fractionation of brain gangliosides. Biochim. Biophys. Acta 296, 160-170.

[15] Zurzolo, C., Lisanti, M.P., Caras, I.W., Nitsch, L. and Rodriguez-Boulan, E. (1993) Glycosylphosphatidylinositol-anchored proteins are preferentially targeted to the basolateral surface in Fischer rat thyroid epithelial cells. J. Cell Biol. 121, 1031-1039.

[16] Prinetti, A., Chigorno, V., Tettamanti, G. and Sonnino, S. (2000) Sphingolipid-enriched membrane domains from rat cerebellar granule cells differentiated in culture. A compositional study. J. Biol. Chem. 275, 11658-11665.

[17] Prinetti, A., Chigorno, V., Prioni, S., Loberto, N., Marano, N., Tettamanti, G. and Sonnino, S. (2001) Changes in the lipid turnover, composition, and organization, as sphingolipid-enriched membrane domains, in rat cerebellar granule cells developing in vitro. J. Biol. Chem. 276, 21136-21145.

[18] Pike, L.J., Han, X., Chung, K.N. and Gross, R.W. (2002) Lipid rafts are enriched in arachidonic acid and plasmenylethanolamine and their composition is independent of caveolin-1 expression: a quantitative electrospray ionization/mass spectrometric analysis. Biochemistry 41, 2075-2088.

[19] Busche, R., Dittmann, J., Meyer zu Duttingdorf, H.D., Glockenthor, U., von Engelhardt, W. and Sallmann, H.P. (2002) Permeability properties of apical and basolateral membranes of the guinea pig caecal and colonic epithelia for short-chain fatty acids. Biochim. Biophys. Acta 20, 55-63. 